\title{
Médicalisation de la société et durabilité des systèmes de santé *
}

Gianfranco Domenighetti **

\section{* Discours officiel proposé lors de la fête inaugurale du $200^{e}$ anniversaire de la société médicale du canton de Berne à Berthoud le 25 octo- bre 2008. \\ ** Le Prof. Gianfranco Domeni- ghetti a été, de 1969 à 2007 directeur des Services de santé du Canton du Tessin et, par conséquent, responsable de la santé publique. Il est professeur d'économie de la santé aux Universités de Lausanne et de la Suisse italienne.}

\section{Correspondance:}

Prof. Gianfranco Domenighetti Università della Svizzera italiana Facoltà di scienze della comunicazione CH-6900 Lugano

\section{gianfranco.domenighetti@} lu.unisi.ch
La thématique de la médicalisation de la vie et de la société remonte au début du siècle passé lorsque la génialité de Jules Romains fait dire au docteur Knock que «les biens portants ne sont que des malades qui s'ignorent», successivement en 1976, lors d'un interview à la revue économique Fortune, Henry Gadsen, CEO de MSD, déclara que son rêve était de produire des médicaments pour les biens portants (rêve aujourd'hui déjà abondamment réalisé), plus récemment un «incipit» d'un article paru sur le British Medical Journal du 13 avril 2002 nous rappelait qu' «on peut faire beaucoup d'argent si l'on arrive a convaincre les bienportants qu'en réalité ils sont des malades». En Suisse, en 1996 déjà, Alex Müller, président de l'Académie Suisse des Sciences Médicales, constatait que «nous souffrons en Suisse d'une surmédicalisation; les contradictions d'une société surmédicalisée rendent nécessaire une redéfinition des objectifs qui passe par la triple révision des pratiques, du développement scientifique et des valeurs de la santé».

Hippocrate semble être peu à peu supplanté par le Docteur Knock, vrai homme d'affaire. Nous sommes probablement en train de vivre la transition depuis la médicine hippocratique vers celle «knockratique». Et voilà comment.

Au delà des causes naturelles (vieillissement démographique) ou exogènes au système de soins (morbidité supplémentaire suite à la croissance de la précarité, de l'insécurité et du stress au travail) qui engendrent une demande supplémentaire de soins qui à son tour pousse à la croissance des coûts sanitaires, la médicalisation de la vie et de la société trouve son fondement dans une extension du domaine de la médicine sur trois niveaux:

\section{Niveau quantitatif}

Du point de vue opérationnel, on arrive à augmenter le nombre potentiel de «malades» par des révisions à la baisse des paramètres qui définissent les seuils du «pathologique» pour toute une série de «facteurs de risque» diffus (notamment hypertension, hypercholestérolémie, diabète, etc.). De ce fait des millions d'individus supplémentaires vont devenir éligibles pour une prise en charge médicalisée en faisant ainsi passer leur statut d'individus subjectivement bien portants«à celui de personnes objectivement malades».

Une étude récente a montrée qu'à l'âge de 40 ans $23 \%$ de la population féminine et $86 \%$ de celle masculine de la Norvège serait, selon les nouveaux seuils d'intervention prévus par les «guidelines» 2003 de la
Société Européenne de Cardiologie, à haut risque cardiovasculaire justifiant ainsi une prise en charge médicalisée (à l'âge de 65 ans $84 \%$ pour les femmes et 92\% pour les hommes).

Il est important de souligner que la médicalisation des facteurs de risque est en train de modifier significativement l'approche thérapeutique qui va de plus en plus se diriger vers le traitement de «probabilités anonymes».

En effet, lorsqu'on traite des individus en bonne santé n'ayant que des facteurs de risque le résultat au niveau individuel n'est mesurable que sur la base de «surrogate endpoints» souvent fallacieux. Une telle médicalisation «épidémique» de masse ne permet pas d'identifier les individus qui auront réellement eu un bénéfice en terme d'événements aigus ou de décès évités.

\section{Niveau temporel}

L'anticipation d'un diagnostic chez des sujets asymptomatiques par la généralisation des «check-up», par la promotion de tests de diagnostique précoce et de dépistages dont l'efficacité est douteuse, controversée ou n'a pas pu être solidement démontrée, constitue très souvent le facteur principal à la base de la médicalisation de la vie, ce qui a fait dire au New York Times du 2 janvier 2007 «What's making us sick is an epidemic of diagnoses».

La diffusion des technologies de diagnostique génétique donnera dans un futur proche à chacun l'opportunité d'être transformé en «malade» juste après la naissance. Il est surprenant de constater l'enthousiasme populaire pour les prestations de diagnostic précoce.

Aux Etats-Unis, par exemple, 50\% des femmes ayant subi l'ablation du col de l'utérus suite à une hystérectomie radicale continuent à faire le PAP-test, en Suisse $60 \%$ de la population serait disposé à se soumettre à un test de dépistage précoce du cancer du pancréas, tumeur pratiquement incurable, toujours en Suisse $65 \%$ des femmes croient que le dépistage par mammographie annule ou réduit le risque de tomber un jour malade d'un cancer du sein.

\section{Niveau qualitatif}

La troisième voie vers la médicalisation est représentée par l'augmentation des conditions et situations propres à la condition humaine qui sont ou qui pourront être transformées en «maladies». Ce n'est donc pas un hasard si le British Medical Journal a déjà publié 
une «classification internationale des non-maladies» comptabilisant plus de 200 conditions réputées à tort comme étant des états morbides.

Paradoxalement les services de santé dépensent des milliards pour lutter contre la maladie alors qu'en réalité ils sont aussi en train de créer des millions de malades supplémentaires et à faire disparaître toute personne en bonne santé. C'est le triomphe de la médicine anticipé il y a presqu'un siècle par Jules Romains.

\section{Le «marché» de la santé}

Moteur de cette dynamique sont les producteurs de technologie médico-sanitaire, c'est à dire l'industrie, dont l'objectif principal (et légitime) est l'élargissement des marchés et l'augmentation des profits et ceci indépendamment de la «plus-value» de l'innovation. Les outils à la base de cette stratégie seront

1. la mise en œuvre de politiques de marketing et de communication, généralement agressives et «nontransparentes», envers surtout les prescripteurs, sans oublier les patients-consommateurs, les financeurs et les régulateurs, et

2. l'utilisation d'incitations économiques et professionnelles souvent "perverses» (mise en situation de conflits d'intérêt, corruption, etc.).

Les facteurs et les conditions sous-jacentes qui favorisent le succès de ces stratégies industrielles seront: la complexité, l'incertitude et l'asymétrie informative généralisée qui caractérisent le «marché» de la santé ainsi que les préférences sociales envers le bien-être et la vie plutôt qu'envers le malaise et la mort.

Ce n'est donc pas un hasard si la diffusion de l' «innovation» technologique a été identifiée par toutes les études économiques comme le principal facteur responsable de la croissance de la dépense sanitaire (au vieillissement démographique on attribue entre 10 et $15 \%$ du taux de croissance).

Si l'on prend en exemple l'industrie pharmaceutique l'on constate notamment que:

- les investissements pour le marketing sont deux fois plus importants que ceux destinés à la recherche et au développement;

- seulement 2,6\% des médicaments mis sur le marché de 1981 à $2005(\mathrm{~N}=3335)$ représentent un progrès thérapeutique majeur ou important $(6,8 \%$ de progrès présent mais limité) et que le $85 \%$ ne sont que des copies de produits existants (à l'exception du prix qui sera multiplié par trois);

- selon Richard Smith, jusqu'à 2005 éditeur du British Medical Journal, les résultats des recherches financées par l'industrie sont presque toujours favorables aux sponsors grâce à toute une série de manipulations des méthodologies d'analyse, et les effets non-désirés très souvent ne sont pas publiés.

Le «marché» de la santé va encore avoir devant lui un bel avenir au moins jusqu'au moment où la richesse nationale, les finances publiques et surtout les revenus des ménages seront en mesure de supporter une charge économique socialisée en croissance libre.

La construction sociale des maladies est en train d'être substituée par la construction industrielle des morbidités. Ceci aura un impact majeur sur l'angoisse individuelle et sociale, sur la croissance de la demande et des coûts et donc sur la durabilité des systèmes de santé «universels» comme nous les connaissons aujourd'hui.

\section{Références}

- Romains J. Knock ou le triomphe de la médecine. Paris: Gallimard; 2000.

- Postel-Vinay N, Corvol P. Le retour du docteur Knock: essai sur le risque cardiovasculaire. Paris: Odile Jacob; 2000.

- Domenighetti G. Grandeur et misère des systèmes universels de santé. Scénario sur la transition du système sanitaire suisse à l'heure de la globalisation. Bull Med Suisses. 2005;84:221-6.

- Le Fanu J. The rise and fall of modern medicine. London: Abacus; 2004.

- Smith R. In search of «non-disease». Br Med J. 2002;324:883-5.

- Moynihan R, et al. Selling sickness: the pharmaceutical industry and disease mongering. Br Med J. 2002;324: 886-91.

- Campbell EG, et al. A national survey of physicianindustry relationships. NEJM. 2007;356:1742-50.

- Angell M. The truth about the drug companies. New York: Random House; 2004

- Smith R. Medical journals are an extension of the marketing arm of pharmaceutical companies. PLos Medicine. 2005;2(5):e138.

- Abramson J, Wright JM. Are lipid lowering guidelines evidence-based? Lancet. 2007;369:168-9.

- Mangin D, et al. Preventive health care in elderly people needs rethinking. Br Med J. 2007;335:285-7.

- Starfield B, et al. The concept of prevention: a good idea gone astray? J Epid Community Health. 2008; 62;580-3.

- Getz L, et al. Estimating the high risk groups for cardiovascular disease in the Norwegian HUNT 2 population according to 2003 European Guidelines: a modelling study. Br Med J. 2005;331:551-6.

- Welch HG. Should I be tested for cancer? Maybe not and here's why. Los Angeles: University of California Press; 2004.

- Domenighetti G, et al. Women's perception of the benefits of mammography screening: populationbased survey in four countries. Int J Epidemiol. 2003; 32:816-21.

- Sirovich BE. Cervical cancer screening among women without a cervix. JAMA. 2004;291:2990-3.

- Hadler NM. The Last Well Person: How to Stay Well Despite the Health-Care System. Montreal: McGill-Queens University Press; 2004. 